\title{
Perfil de pacientes com câncer ginecológico em tratamento Quimioterápico
}

\author{
Profile of patients with gynecological cancer under chemotherapy \\ Perfil de pacientes con cáncer ginecológico bajo tratamiento Quimioterápico
}

\section{Elisângela Maria Soares', Sueli Riul da Silva'}

'Universidade Federal do Triângulo Mineiro. Curso de Graduação em Enfermagem. Uberaba, MG

Submissão: 01/07/2007

Aprovação: 12/12/2009

\section{RESUMO}

Estudo do perfil sócio-epidemiológico demográfico das pacientes em tratamento Quimioterápico por câncer na Enfermaria de Ginecologia e Obstetrícia do HE-UFTM, apresentado no período de 1998 a 2006. Estudo retrospectivo cuja coleta de dados foi realizada a partir de dados existentes no prontuário. Foi construído banco de dados no programa Epi-info, utilizando-se para análise distribuição simples, mediana com valores entre máxima e mínima e desvio padrão. A amostra foi constituída por $32 \mathrm{I}$ porntuários. Os dados dos pacientes revelaram faixa etária mais incidente 46 a 50 anos, $45 \%$ dos casos de câncer foi de mama, o Quimioterápico mais utilizado foi ciclofosfamida em 173 tratamentos. Houve aumento na freeuência do câncer de mama. Conclui-se Que houve mudança no perfil das pacientes.

Descritores: Câncer/Quimioterapia; Saúde da mulher; Enfermagem.

\section{ABSTRACT}

A study of socio-epimiological demographic profile of patients in chemotherapy for cancer in the Gynecology and Obstetrical Ward in HE- UFTM, presented in the periodo from 1998 to 2006. Retrospective study whose data collection was obtained from health records It was built a data base using Epi-Info. Data analysis considered simple distribution, mediacy with value between the maximum and the minimum and stand deviation. The sample was constituted of $32 \mathrm{I}$ health records; the more incident age group was 46 to 50 years-old; breast cancer was the most noticed cancer (45\%); the chemotherapy most used was ciclofosfamida, in 173 treatments; had an increase in breast cancer. It was concluded that had a change in the patient's profile.

Descriptors: Neoplasms/chemotherapy; Women's health; Nursing.

\section{RESUMEN}

Estudio del perfil socio-epidemiológico demográfico de las pacientes en tratamiento Quimioterapico por cáncer en la Enfermería de Ginecología y Obstetricia del HE-UFTM, presentado en lo periodo de 1998 al 2006. Estudio retrospectivo cuya recolleción de datos, fue realizada en el prontuario. Fue construido banco de dados en el programa Epi-info, se utilizó para analice distribución simple, mediana con valores entre máxima y mínima y desvío padrón. La muestra fue constituida de 321 prontuarios. La faja de edad los pacientes más incidente fue de 46 a 50 años, $45 \%$ de los casos de cáncer fue el de mama, el Quimioterapico mas utilizado fue ciclofosfamida, en 173 tratamientos; hubo aumento en la frecuencia del cáncer de mama. Se concluyó que hubo un cambio en el perfil de las pacientes.

Descriptores: Neoplasmas/Quimioterapia; Salud de la mujer; Enfermería. 


\section{INTRODUÇÃO}

A palavra câncer deriva do latim e significa caranguejo, sendo possivelmente utilizada devida sua adesão aos tecidos vivos ${ }^{(1)}$. Atualmente câncer denomina todo tumor maligno. O câncer é um distúrbio genético no Qual se perde o controle normal do crescimento celular. Normalmente, ao longo da nossa vida, a reprodução celular na maioria dos tecidos é controlada por mecanismos biológicos intrínsecos. No câncer, esse mecanismo de controle é perdido ${ }^{(2)}$.

O câncer é a segunda causa de morte por doença no Brasil o Que se configura como um problema de saúde pública tanto nos países desenvolvidos como nos países em desenvolvimento por demandar ações com variados graus de complexidade. No sexo feminino, a neoplasia maligna de mama é mais incidente, seguindose a de pele não melanoma e a de colo de útero. De maneira geral, as regiões Sudeste e Sul apresentam as taxas mais elevadas, em ambos os sexos ${ }^{(3)}$

As duas últimas décadas testemunharam um considerável avanço no tratamento do câncer, tendo-se a cura como objetivo terapêutico real em $50 \%$ dos tumores diagnosticados. São as principais formas de tratamento do câncer: cirurgia, radioterapia, Quimioterapia, hormonioterapia e a terapia combinada Que pode ser a combinação de todas as outras ${ }^{(3)}$.

A quimioterapia antiblástica consiste no emprego de substâncias Químicas, isoladas ou em combinação, com o objetivo de tratar as neoplasias malignas. É o tratamento de escolha para doenças malignas do sistema hematopoético e para os tumores sólidos, Que apresentam metástases regionais ou à distância. A maioria dos agentes antiblásticos atua de forma não específica, lesando tanto células malignas Quanto benignas ${ }^{(4)}$.

Os antineoplásicos são classificados de duas maneiras principais: de acordo com sua estrutura Química e função em nível celular e com a especificidade no ciclo celular. A primeira divide-os em seis grupos: agentes alQuilantes (ciclofosfamida, cisplastina e carboplatina); antimetabólitos (metrotexato e mercaptopurina); antibióticos antitumorais (doxorrubicina e bleomicina); plantas alcalóides (vincristina e paclitaxel); agentes múltiplos (hidroxiuréia) e hormônios e antagonistas hormonais (estrogênios e antiestrogênios) $)^{(4)}$.

Hoje se sabe Que a utilização da polieuimioterapia (utilização de mais de uma agente citostático em combinação) tem proporcionado vantagens no tratamento oncológico, pois proporciona sinergismo-somatório dos efeitos isolados; retardam a resistência tumoral por utilizar-se drogas com mecanismos de ação diferentes e doses menores diminuindo assim os efeitos colaterais $^{(4)}$.

Outra forma de tratamento é a radioterapia Que constitui uma forma de tratamento localizado, com utilização de radiação ionizante, proveniente de aparelhos ou radioisótopos naturais ou artificiais. Essa forma de tratamento objetiva destruir as células tumorais da região comprometida, contudo tampouco é seletiva. Pode agir por efeito direto da radiação (átomos sofrem excitação e ionização o Que desencadeia uma mudança biológica) e corresponde a 30\% de sua ação ou então agir levando ao efeito indireto (em Que moléculas de água se transformam em radicais livres) correspondendo a $70 \%$ de seu efeito ${ }^{(5)}$. A radiação pode ser administrada sob duas formas, teleterapia e braQuiterapia. $\mathrm{Na}$ teleterapia os aparelhos emissores estão distantes alguns centímetros do paciente, já na braQuiterapia as fontes de radiação são colocadas em contato direto com o tumor ${ }^{(1)}$.

A cirurgia ainda é uma das formas mais freeüentes e adequadas de tratamento para a maioria das neoplasias malignas. A cirurgia oncológica despende cuidados próprios tais como incisão cirúrgica ampla e adequada, proteção da ferida operatória para impedir o implante de células tumorais, nas cavidades deve ser realizado inventário minucioso das víceras, estadiamento correto, cuidado para não violar a integridade tumoral, margem de segurança e troca de luvas após a ressecção tumoral ${ }^{(5)}$.

A hormônioterapia envolve a manipulação de hormônios com o objetivo de deter o crescimento tumoral e pode ser realizada de Quatro maneiras: terapêutica aditiva, ablática, competitiva e inibitória ${ }^{(5)}$.

O estadiamento é realizado ao se avaliar a extensão da doença no organismo (órgão de origem, órgãos ou estruturas adjacentes, linfonodos regionais e a distância). Existem vários sistemas de estadiamentos do câncer sendo o mais usado o TNM Que se baseia na pesquisa de três componentes: T-extensão do tumor primário; $\mathrm{N}$-ausência ou presença de metástase em linfonodos regionais e M-ausência ou presença de metástase à distância. Para câncer ginecológico se utiliza também o sistema FIGO (Federação Internacional de Ginecologia e Obstetrícia) ${ }^{(1)}$.

Os cânceres mesmo não apresentando causas definidas podem apresentar fatores de risco. Para o câncer de mama os fatores de risco incluem história familiar de câncer de mama, diagnóstico confirmado de hiperplasia atípica, densidade da mama aumentada, história de menarca precoce ou menopausa tardia, obesidade após a menopausa, uso de contraceptivos orais ou reposição de hormônios orais (estrogênio e progesterona) pós-menopausa, nuliparidade ou primeira gravidez após 30 anos de idade e consumo de bebidas alcoólicas ${ }^{(6)}$.

Para o câncer de colo de útero, estudos epidemiológicos e de história natural da doença mostram uma associação causal entre a infecção pelo vírus papiloma humano, além disso, os principais cofatores são história de infecções sexualmente transmissíveis, início precoce da atividade sexual, multiparidade, uso de contraceptivos orais e tabagismo ${ }^{(7)}$. As taxas de incidência de lesões pré-invasivas do câncer do colo uterino, moderadas e graves, têm aumentado nas mulheres mais jovens ${ }^{(8)}$.

Hoje evidenciamos a alta incidência de câncer na população feminina o Que demonstra déficit nas ações de prevenção, de diagnóstico precoce, aumento da idade média dos indivíduos, aumento nos fatores de risco e mudanças de estilo de vida. O câncer é relatado com uma "sentença de morte" muitas das vezes, estando associado a dor, sofrimento e degradação $0^{(9)}$.

Com a mudança do perfil sócio epidemiológico das pacientes com câncer e as novas possibilidades terapêuticas observadas nos últimos anos tem se evidenciado uma sobrevida maior das pacientes, o que nos faz refletir sobre a necessidade de melhoria da Qualidade de vida, assim como melhoria na assistência prestada por profissionais na área da oncologia. A Organização Mundial de Saúde engloba em seu conceito de Qualidade de vida a interação do individuo com seu ambiente interno e externo, "Qualidade de vida é a percepção de uma pessoa de sua posição na vida no contexto 
da cultura e sistema de valores nos Quais ele vive e em relação aos seus objetivos, expectativas, padrões e preocupações"(10).

Partindo-se das reflexões da alta incidência e da mudança do perfil das pacientes com câncer ginecológico no Brasil, Questionamos o percentual de mulheres Que realizam tratamento do câncer na Enfermaria de Ginecologia e Obstetrícia do Hospital Escola da Universidade Federal do Triângulo Mineiro - EGO/HE/UFTM, assim como o perfil sócio-epidemiológico demográfico por elas apresentado nos últimos oito anos (1998/2006).

Desta forma, delineamos o seguinte objetivo para este estudo: descrever as variações existentes, entre os anos 1998-2006, no perfil sócio-epidemiológico e demográfico das pacientes, de forma Que possamos propor ações de prevenção ou intervenções baseadas no perfil observado nas pacientes, estabelecendo dessa forma um tratamento planejado e Que possibilite ações de rápida intervenção e mais eficazes. Para melhor descrever este estudo transcrevemos o objetivo em Quatro objetivos específicos:

- descrever características sócio-demográficas e epidemiológicas das pacientes em tratamento Quimioterápico para câncer ginecológico no HE da UFTM, no período de 1998 a 2006;

- descrever o número de casos dos cânceres ginecológicos nos últimos oito anos, entre as pacientes Que realizaram tratamento Quimioterápico no HE da UFTM;

- verificar a incidência dos tipos de câncer ginecológico, de acordo com a faixa etária e o período de maior ocorrência;

- descrever os tipos de Quimioterápicos antineoplásicos utilizados para tratamento de câncer ginecológico, entre os anos de 1998 a 2006.

\section{MÉTODOS}

Trata-se de um estudo retrospectivo realizado no Hospital Escola da UFTM, com informações relativas ao período de 1998 a 2006 de pacientes portadoras de câncer ginecológico em tratamento Quimioterápico na instituição.

Os dados foram coletados nos prontuários das pacientes. Para tanto as pacientes foram identificadas na agenda de Quimioterapia preenchida no serviço.

O instrumento de coleta de dados (apêndice um) contempla as variáveis sócio-demográficas: idade, procedência, naturalidade, e epidemiológicas: tipo de câncer; tratamento utilizado e protocolo Quimioterápico. Os dados foram coletados após aprovação do Comitê de Ética em Pesquisa com Seres Humanos da Universidade Federal do Triângulo Mineiro.

Foi construído banco de dados no programa Epi- info, utilizandose para análise dos objetivos, distribuição simples, mediana com valores entre máxima e mínima e desvio padrão. Para a análise das variáveis relacionadas à faixa etária foram utilizados recortes de cinco anos com o intuito de se observar mais facilmente as tendências.

\section{RESULTADOS E DISCUSSÃO}

O grupo de pacientes do estudo é composto por 321 sujeitos em acompanhamento no serviço de Quimioterapia, sendo todos do sexo feminino, com idade média de 50,73 anos, variando entre 16 e 84 anos, o desvio padrão 14,478, mediana de 51 anos, onde $25 \%$ tem até 42 anos e $75 \%$ até 61 anos. Ao se agrupar as idades por faixa etária obtivemos como faixa etária mais incidente 46 a 50 anos (15,99\%), seguida por 56 a 60 anos com 13,48\%.

Quanto à naturalidade obtivemos 258 respostas $(80,4 \%$ do total da pesquisa). Sendo Que destas $24,03 \%$ são naturais de Uberaba, 5,43\% de Sacramento e Araxá respectivamente e os restantes estão distribuídos entre outras 100 cidades. Procedem de Uberaba $66,9 \%$.

O principal tipo de câncer tratado nos anos de 1998 a 2006 na EGO-HE-UFTM foi o de mama com 144 tratamentos Que correspondem a $45 \%$ dos casos acompanhados. O câncer de mama foi o de maior incidência entre as mulheres em 2001 no Brasil, segundo estimativas do Ministério da Saúde ${ }^{(3)}$, em 2003 foram 41.610 novos casos, ou seja, 46 casos/100.000 mulheres, com 9.335 óbitos. A taxa de óbito, Que no ano de 1979 era de aproximadamente 5,77/100.000 mulheres, subiu para 9,7/ 100.000 mulheres em $1998^{(1)}$. Constitui-se na primeira causa de morte, por câncer, entre as mulheres, registrando-se uma variação percentual relativa de mais de $80 \%^{(12)}$.

As taxas de incidência de câncer de mama aumentam com a idade, alcançando seu pico na faixa etária de 65 a $70 \operatorname{anos}^{(6)}$. No Brasil para o ano de 2006 a maior taxa bruta de incidência por 100.000 mulheres segundo a Unidade da Federação (neoplasia maligna da mama feminina) é do Estado do Rio de Janeiro com 96,95 e a menor taxa corresponde ao estado do Maranhão com 9,54 sendo Que no estado de Minas Gerais é de 42,82 casos. No Brasil a incidência prevista para o ano de 2006 de câncer de mama é de 48.930 casos $^{(13)}$. Mesmo com sua significativa incidência o câncer de mama é um tipo de câncer Que possibilita sobrevida Quando detectado precocemente $\mathrm{e}^{(14)}$.

No HE 98 casos $(30,6 \%)$ são de câncer de colo uterino. O Que condiz com dados da bibliografia sendo este o segundo tipo de câncer em incidência. No Brasil, segundo Instituto Nacional do Câncer-INCA, em 2003 houve cerca de 16.000 novos casos, com a morte de cerca de 4.000 mulheres. A incidência de câncer de colo de útero torna-se evidente na faixa etária de 20 a 29 anos e o risco aumenta rapidamente até atingir seu pico geralmente na faixa etária de 45 a 49 anos. Quase $80 \%$ dos casos novos ocorrem em países em desenvolvimento onde, em algumas regiões, é o câncer mais comum entre as mulheres ${ }^{(6)}$.

No Brasil a incidência de câncer de colo uterino esperada para o ano de 2006 segundo dados do INCA em estudo por 100.000 mulheres, estimada segundo a Unidade da Federação (neoplasia maligna de colo de útero), é Que o estado de maior incidência seja o do Rio Grande do Sul com 30,9 casos, já no estado de Minas Gerais é de 14,47 e o de menor incidência é a Paraíba com 8,4 casos. No Brasil a incidência é de $19.260 \operatorname{casos}^{(13)}$.

O câncer de ovário foi encontrado em 48 casos (15\%), 2 I casos com outro tipo de patologia $(6,6 \%)$, cinco casos de câncer de endométrio $(1,6 \%)$ e dois casos de câncer de vagina e vulva respectivamente $(0,6 \%)$. Esta informação condiz com o apresentado pela literatura Que demonstra uma maior incidência no Brasil de câncer de mama, com risco estimado para o ano de 2006 de 52 casos para 100 mil mulheres, seguido pelo de colo uterino com 20 casos para $100 \mathrm{mil}^{(13)}$.

São cânceres ginecológicos, de menor incidência o câncer de vulva, de vagina, de endométrio e de ovário. O câncer de ovário é 
considerado como o mais agressivo de todos os tumores ginecológicos, atinge mulheres com mais de 40 anos sendo seu pico de incidência entre 75 e 79, mostrando Que sua incidência cresce com a idade ${ }^{(15)}$. O câncer de endométrio é o câncer ginecológico mais comum nos países desenvolvidos e possui uma relativa baixa mortalidade pelo fato deles estarem confinados no útero no momento do diagnóstico, a média de idade de acometimento é de 60 anos de idade. $O$ de vagina é também raro representando cerca de $1 \%$ a $2 \%$. O câncer de vulva é considerado como de ocorrência rara, respondendo por $4 \%$ a $5 \%$ de todos os cânceres ginecológicos.

Segundo Documento de Consenso do Ministério da Saúde o Brasil diferentemente de países desenvolvidos como Estados Unidos, Canadá, Reino Unido, entre outros, está apresentando um aumento da incidência de câncer acompanhado do aumento da mortalidade, o Que segundo o documento pode ser atribuído a um retardamento do diagnóstico e da instituição terapêutica adequada ${ }^{(16)}$.

Ao se observar a faixa etária das mulheres nos diversos tipos de câncer estudados foi observado: colo uterino 14 casos (14,4\%) na faixa etária de 46 a 50 anos (Figura I), o Que condiz com dados da literatura Que refere maior incidência na faixa etária de 40 a 50 $\operatorname{anos}^{(1)}$. As taxas de incidência de lesão pré-invasora do câncer de colo tem aumentado nas mulheres mais jovens, podendo-se pressupor Que tais resultados ocorram devido ao inicio precoce da atividade sexual ${ }^{(17)}$.

O câncer de mama, 27 casos (18,88\%) ocorreu entre 41 a 45 anos, seguido por $18,18 \%$ entre 46 a 50 anos. Segundo dados do INCA o câncer de mama possui como faixa etária mais incidente 40-69 anos, possuem o pior prognóstico o grupo de mulheres jovens (idade igual ou inferior a 35 anos) Que desenvolvem um câncer de mama, e também aQuelas cujo diagnóstico venha a ser estabelecido a partir dos 75 anos de idade ${ }^{(18)}$. Ainda, 20,83\% (10 casos) apresentam câncer de ovário na faixa etária de 51 a 55 anos, comum para tumores epiteliais.

Para o câncer de endométrio 20\% estão na faixa etária de 46 a 50,56 a 60,61 a 65 e 71 a 75 anos respectivamente o Que corresponde a um caso por faixa etária, sendo Que na literatura a maior incidência está entre 60-70 anos.

Quanto ao câncer de vulva e vagina foram observados dois casos para cada neoplasia, de 31 a 35 e de 36 a 40 anos para o câncer de vulva, o que difere da literatura Que demonstra maior incidência acima de 50 anos, tendo que se considerar o pequeno número de sujeitos da pesquisa apresentando essa patologia, e de 46 a 50 e 61 a 65 anos para o câncer de vagina considerado o mais raro dos cânceres ginecológicos.

No que se refere a outros tipos de neoplasia Que necessitam de tratamento Quimioterápico houveram sete casos $(33,33 \%)$ na faixa etária de 16 a 20 anos. A mola hidatiforme representa 14 casos (gráf. 2). A literatura evidência uma maior freeüência na terceira década, porém, demonstram Que ao se comparar o número de molas hidatiformes e o número de gravidezes por faixa etária, a incidência é maior nas adolescentes e em mulheres acima de 40 $\operatorname{anos}^{(19)}$.

No Brasil registrou-se um aumento nos últimos cinco anos, no número de pacientes oncológicos atendidos pelas unidades do SUS $^{(13)}$. No HE da UFTM durante o período de realização do estudo ao se analisar o tipo de câncer e anos de maior incidência, observamos que 15 casos $(15,63 \%)$ dos cânceres de colo de útero ocorreram em 2002; o câncer de mama, 23 casos (16,0\%), no ano de 2006 observando-se um crescimento do número de casos

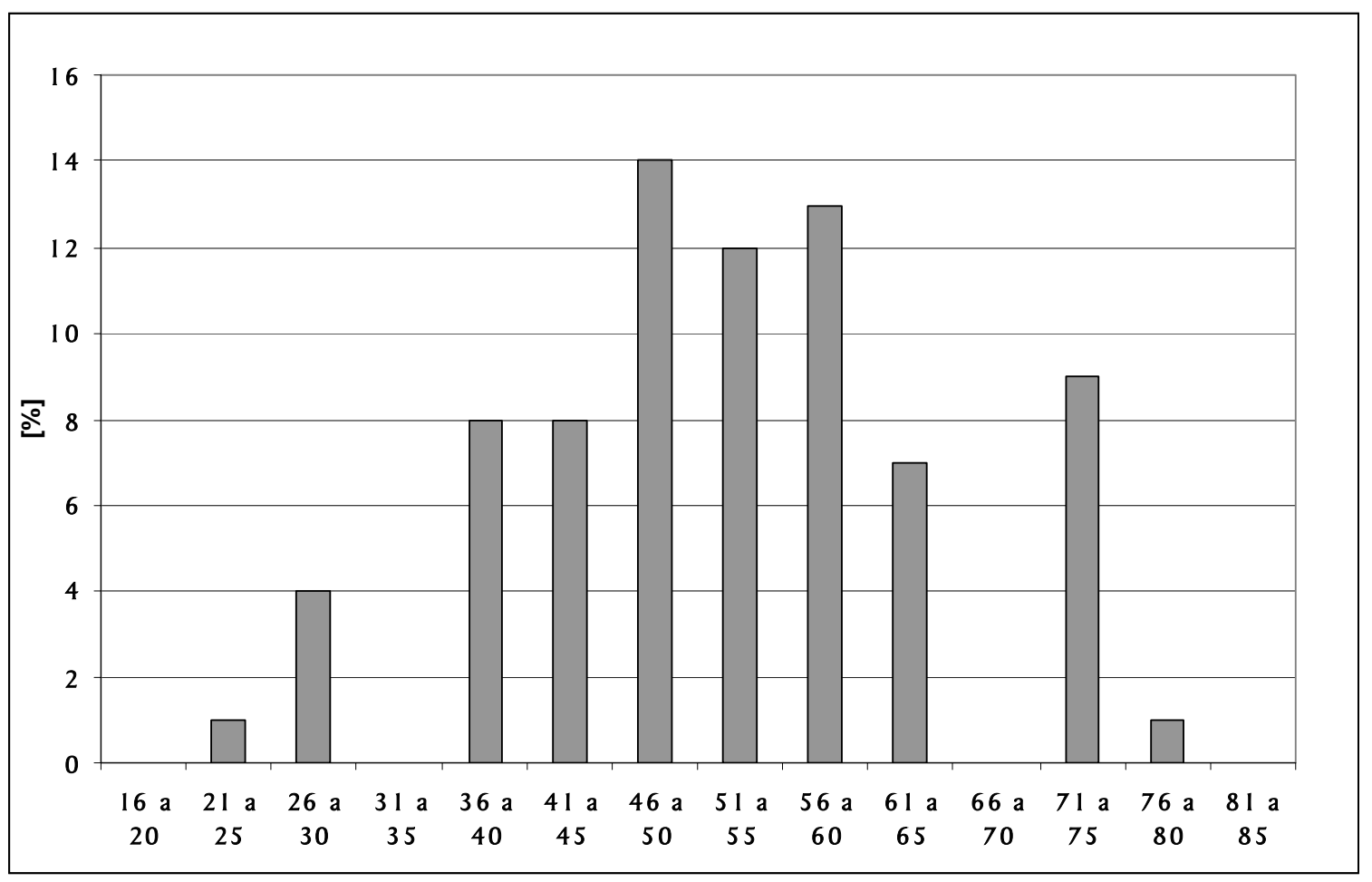

Figura 1. Casos de câncer de colo uterino por faixa etária. 
de câncer de mama no período do estudo, Quando o ano de menor índice foi 1998 com 10 casos, o Que vem de encontro com dados dos Registros de Base Populacional do INCA. O INCA refere um crescimento contínuo, o Que segundo esse instituto pode ser resultado de mudanças sócio-demográficas e de acessibilidades da população aos serviços ${ }^{(13)}$.

O câncer de ovário apresentou incidência de nove casos ( $18,75 \%)$ no ano de 2002; câncer de endométrio, dois casos (40\%), no ano de 2002; vagina dois casos durante o período de estudo sendo um no ano de 1998 e um em 2002; para outras patologias Que necessitam de tratamento Quimioterápico a maior incidência foi em 2004 com cinco casos $(23,80 \%)$ e o câncer de vulva assim como o de vagina também apresentou durante o período de estudo dois casos, um em 2003 e outro em 2005.

No Que se refere à data do início do tratamento o ano em Que se teve maior índice de admissão foi o de 2002 com 48 tratamentos iniciados, 15,05\% do total entre os anos de 1998 a 2006. Com uma média de atendimento por ano de 35,4 clientes.

Todos os sujeitos da pesquisa utilizaram Quimioterapia, sendo Que 187 casos $(58,26 \%)$ realizaram em conjunto pelo menos um procedimento cirúrgico e/ou radioterapia Que foi realizada por 156 pacientes (48,6\% dos casos).

No que se refere aos Quimioterápicos utilizados, a ciclofosfamida no período de realização da pesQuisa foi o mais utilizado- 173 tratamentos (53,9\% dos casos), é indicado em ginecologia para tratamento de adenocarcinoma de ovário, carcinoma de ovário e mama. ${ }^{(4)}$ No HE desde o ano de 1998 é empregado, sendo o maior número de vezes em 2004 com 26 tratamentos. A cisplastina utilizada de forma mais comum em tumores de ovário e também em câncer de mama ${ }^{(4)}$, empregada em 132 tratamentos $(41,12 \%$ dos casos) desde 1998 com seu pico de utilização em 2002 e 2004 em 18 tratamentos. Epirrubicina, 10 I tratamentos (3 1,46\%) indicada principalmente para tratamento de câncer de mama ${ }^{(4)}$ desde 1998 é utilizada no HE tendo pico em 2006 - 19 tratamentos. Etoposide, 83 tratamentos $(25,85 \%)$ além de suas indicações mais comuns como câncer de colo uterino é também utilizado para o tratamento de câncer de mama, ovário e neoplasias trofoblásticas e de células germinativas ${ }^{(4)}$ com predomínio de uso em 2002- 16 tratamentos; bleomicina, 81 tratamentos $(25,23 \%)$ possui como indicações para uso tratamentos de câncer de colo uterino e vulva ${ }^{(4)}$ utilizada em maior número de vezes nos anos de 1998 e 2002-13 casos.

A droga 5-fluoro-uracil com 79 tratamentos (24.61\%) indicada para tratamento de câncer de mama, útero e ovário ${ }^{(4)}$ apresentou Queda na utilização durante o período da pesQuisa, com um máximo de 16 utilizações no ano de 1999 e um mínimo em 2006 com uma. A adriamicina foi utilizada 48 vezes (14,95\%) no período de 1999 a 2006 tendo sido empregada um maior número de vezes em 2004-nove tratamentos. Methotrexato, 38 tratamentos $(11,84 \%)$ sendo Que dentre suas utilizações estão principalmente o tratamento de mola hidatiforme e câncer de mama ${ }^{(4)}$ sendo também utilizado no tratamento do câncer de ovário e mais empregado no ano de 1999. Paclitaxel 33 tratamentos (10,28\%), droga indicada para o tratamento de carcinoma de ovário e de mama avançado ${ }^{(4)}$ utilizada durante todo o período da pesquisa tendo seu máximo, nove tratamentos em 2006. Carboplatina, 19 tratamentos (5,92\%), indicada principalmente em carcinoma avançado de ovário de origem epitelial e menos comumente em câncer de mama e endométrio, utilizada de 1998 a 2006 sendo mais empregada em $2002 \mathrm{em}$ seis tratamentos. A vincristina, utilizada em três tratamentos $(0,93 \%)$ indicado no tratamento de câncer de mama, ovário, sarcoma de tecidos moles e neoplasias trofoblásticas ${ }^{(4)}$ foi utilizada nos anos de 2000 em um tratamento e 2002 em dois. Melfalan dois tratamentos $(0,62 \%)$ indicado no tratamento de câncer de mama e ovário avançados e trofoblásticos, utilizado no ano de 2002 em dois tratamentos.

Com relação aos procedimentos cirúrgicos, foram realizados: Quadrantectomia em 52 pacientes, mastectomia em 29, panhisterectomia em 14, curetagem em nove, histerectomia em seis, ooforectomia e laparotomia em dois casos (gráf. 8). Nas três últimas décadas, a realização de cirurgias radicais (mastectomias) vem sendo reduzida gradativamente em favor das cirurgias conservadoras (segmentectomias), sendo um fator determinante para o tratamento conservador o tamanho do tumor ${ }^{(1)}$.

A análise dos dados apresentados colocou em Questão a causa do aumento da realização das cirurgias conservadoras no HE da UFTM durante o período de 1998 a 2006. As cirurgias conservadoras são indicadas segundo Documento de Consenso para o Controle de Câncer de Mama, Quando o diâmetro tumoral é menor Que três centímetros, entre outros pré-requisitos ${ }^{(16)}$. Em estudo realizado no Hospital do Câncer do INCA, dados de 1998 apresentam o estadiamento III para o câncer de mama com $44,8 \%$, o IV com 16,3\%, enquanto apenas 6,3\% tiveram o seu diagnóstico nos estádios 0 e I. A prevalência de cirurgias conservadoras no tratamento do câncer de mama no HE da UFTM pode estar relacionada a um bom rastreamento evidenciando alterações previamente desconhecidas ou estar relacionado a forma relativamente lenta de evolução desse tumor.

lá a radioterapia é um tratamento de ampla utilização. Dos tumores malignos mais de $60 \%$ terão indicação de irradiação no curso da evolução $0^{(12)}$

\section{CONCLUSÃO}

Ao término do estudo, pôde-se observar parte do perfil sóciodemográfico e epidemiológico das pacientes que realizam tratamento Quimioterápico na EGO/HE/UFTM. Dentre as observações pôde-se destacar:

- realizaram tratamento no período do estudo (1998 a 2006)

32 I sujeitos;

- a faixa etária mais incidente foi de 46 a 50 anos;

- são naturais de Uberaba 24,3\% dos sujeitos;

- são procedentes de Uberaba 66,9\%;

- o maior índice de admissão foi o ano de 2002 com 48 tratamentos/ano;

- a média de atendimentos/ano no período do estudo foi de 35,4 clientes/ ano;

- o câncer mais incidente foi o de mama com 144 tratamentos (45\% dos casos);

- o Quimioterápico mais utilizado foi a ciclofosfamida, utilizada em 173 tratamentos;

- a faixa etária das pacientes em tratamento, para o câncer de colo uterino, $14,43 \%$ dos casos, foi de 46 a 50 anos, para o câncer de mama, 18,88\%, 41 a 45 anos e para o câncer de 
ovário, 20,83\%, na faixa etária de 51 a 55 anos;

- houve um aumento na incidência do câncer de mama no período do estudo, com 10 casos no ano de 1998 e 23 casos no ano de 2006;

- houve decréscimo na utilização de 5-fluoracil e crescimento na utilização de paclitaxel.
Acompanhar o número e a evolução dos casos tratados em uma localidade permite Que seja definido um perfil dos clientes em tratamento e Que apresentemos a real necessidade de uma implementação eficaz de atendimentos básicos de saúde, isso com o intuito de Que sejam adotadas medidas preventivas e realizados diagnósticos precoces permitindo, assim, maior possibilidade de cura.

\section{REFERÊNCIAS}

1. Ayoub AC, Fontes ALC, Silva MAA, Alves NRC, Gigliotte P, Silva YB. Planejando o cuidar na enfermagem oncológica. São Paulo: Lemar; 2000.

2. Weinberg RA. How cancer arises. In: Freeman WH. What you need to know about cancer: a special issue. New York: Scientific American; 1997. p. 3-14.

3. Ministério da Saúde (BR). Instituto Nacional do Câncer. Ações de enfermagem no controle do câncer. $2^{\mathrm{a}}$ ed. Rio de Janeiro: INCA; 2002.

4. Bonassa EMA. Enfermagem em terapêutica oncológica. $3^{\mathrm{a}}$ ed. São Paulo: Atheneu; 2005.

5. Forones NM, Garcia Filho RJ, Tadokoro H, Freire CAR. Guias de medicina ambulatorial e hospitalar - oncologia. Barueri: Manole; 2005.

6. Ministério da Saúde (BR). Instituto Nacional do Câncer. Câncer no Brasil: dados dos registros de câncer de base populacional. Rio de Janeiro(RI): INCA; 2003. [citado em 15 jan 2007]. Disponível em: http://www.inca.gov.br/regpop/2003.

7. Guarisi R, Hardy E, Derchain SFM, Carvasn GAF, Borges JBR. Rastreamento, diagnóstico e tratamento das lesões precursoras e do câncer invasor de colo uterino no Município de Franco da Rocha, SP. Rev Bras Cancerol 2004; 50(1): 7-15.

8. Ferreira MLM, Oliveira C. Conhecimento e significado para funcionárias de industrias têxteis sobre prevenção do câncer do colo-uterino e deteç̧ão precoce do câncer de mama. Rev Bras Cancerol 2006; 52(1): 5-15.

9. Gazzi G, Kajika M, Rodrigues C. O paciente com câncer: crenças e sentimentos sobre sua doença e o tratamento. Acta Oncol Bras 1991; 1 I(1/3): 123-6.

10. Fleck MPA, Leal OF, Louzada S, Xavier M, Chachamovich E, Vieira G, et al. Desenvolvimento da versão em português do instrumento de avaliação de Qualidade de vida da OMS (WHOQOL-100). Rev Bras Psieuiatr 1999; 2 I(1): 19-28.

11. Ministério da Saúde (BR). Instituto Nacional do Câncer. Prevenção e controle de câncer-normas e recomendações do INCA. Rev Bras Cancerol 2002; 48(3): 317-32.

12. Ministério da Saúde (BR). Instituto Nacional do Câncer. Falando sobre câncer de mama. Rio de Janeiro(RI): INCA; 2005. [citado em 15 jan 2007]. Disponível em: http://www.inca.gov.br/ conteudo/.

13. Ministério da Saúde (BR). Instituto Nacional do Câncer. Registro de câncer de base populacional. Rio de laneiro(RI): INCA; 2006. [citado em 15 jan 2007]. Disponível em: http://www.inca.gov.br/ regpop/2006.

14. Haber S, Ayres L, Goodheart C, Lubin L, Siegel M, Acuff C, et al. Breast cancer: a psychological treatment manual. New York: Springer; 1995.

15. Lopes ERL, Rebelo MS, Abib AR, Abreu E. Câncer de mama: epidemiologia e grupos de risco. Rev Bras Cancerol 1996; 42(2): $105-16$.

16. Ministério da Saúde (BR). Controle do câncer de mamadocumento de consenso. Rev Bras Cancerol 2004; 50(2): 77.

17. Zeferino LC, Costa AM, Panetta K, Jorge JPN. Screening da neoplasia cervical. I Bras Ginecol 1996; 106(1 I-12): 415-9.

18. Santi M, Capocaccia R, Verdecchia A, Esteve J,Gatta G, Micheli A, et al. Survival of women with breast cancer in Europe: variation with age, year of diagnosis and country. Int I Cancer 1998; 77(5): 679-83.

19. Yazaki-Sun S, Monteleone PPR, Bertini AM, Camano L. A importância da idade da paciente no prognóstico da mola hidatiforme. Ars Cvrandi 1992; 25(7): 34-8. 